\title{
Diffusion tensor changes in patients with amnesic mild cognitive impairment and various dementias
}

\author{
Ta-Fu Chen ${ }^{\mathrm{a}}$, Chih-Chun Lin ${ }^{\mathrm{a}, 1}$, Ya-Fang Chen ${ }^{\mathrm{b}, *}$, Hon-Man Liu ${ }^{\mathrm{b}}$, Mau-Sun Hua ${ }^{\mathrm{c}}$, \\ Yung-Chien Huang ${ }^{\mathrm{e}}$, Ming-Jang Chiu ${ }^{\mathrm{a}, \mathrm{d}, *}$
}

a Department of Neurology, National Taiwan University Hospital, College of Medicine, Taipei, Taiwan

${ }^{b}$ Department of Medical Imaging, National Taiwan University Hospital, College of Medicine, Taipei, Taiwan

${ }^{\mathrm{c}}$ Department of Psychology, National Taiwan University, Taipei, Taiwan

${ }^{\mathrm{d}}$ Neurobiology and Cognitive Science Center, National Taiwan University, Taipei, Taiwan;

e Department of Radiology, Cathay General Hospital, Taipei, Taiwan

\section{A R T I C L E I N F O}

\section{Article history:}

Received 15 November 2007

Received in revised form 21 June 2008

Accepted 9 September 2008

\section{Keywords:}

Alzheimer's disease

Subcortical ischemic vascular dementia

Frontotemporal dementia

Amnesic mild cognitive impairment

Diffusion tensor imaging

Magnetic resonance imaging

\begin{abstract}
A B S T R A C T
White matter damage and its contribution to clinical manifestations in patients with dementia have been increasingly recognized. To explore white matter changes in different types of dementia, we examined brain water diffusivity with diffusion tensor imaging (DTI). We measured fractional anisotropy and mean diffusivity of multiple white matter regions in patients with amnesic mild cognitive impairment (MCI, $n=10$ ), Alzheimer's disease (AD, $n=30$ ), subcortical ischemic vascular dementia (SIVD, $n=18$ ), frontotemporal dementia (FTD, $n=7)$, and control subjects $(n=20)$. We performed pairwise comparisons in each region of interest between patients and controls. MCI patients showed diffusion tensor change (DTC) in the left anterior periventricular (PV) area, possibly in the right posterior PV area, and the genu of the corpus callosum. AD patients showed DTC in the corpus callosum, and in frontal and parieto-occipital subcortical and anterior PV areas. In SIVD patients, DTC occurred in the genu of the corpus callosum, and in bilateral frontal subcortical and PV areas. FTD patients differed from controls in showing DTC in the temporal and frontal subcortical areas, the genu of the corpus callosum and PV areas. The degree of DTC correlated with the clinical severity of dementia as assessed by the clinical dementia rating (CDR). Mean diffusivity was diffusely and positively associated with the CDR scores. Fractional anisotropy of the PV areas was negatively associated with the CDR scores, suggesting a critical role of the lateral cholinergic pathways.
\end{abstract}

(c) 2008 Elsevier Ireland Ltd. All rights reserved.

\section{Introduction}

Pathological characteristics of Alzheimer's disease (AD) are conventionally noted as cortical involvement with neurofibrillary tangles and senile plaques (Arnold et al., 1991). However, white matter damage and its contribution to clinical manifestations in patients with dementia have been increasingly recognized. Decline in intelligence is associated with changes in white matter volume (Garde et al., 2005). White matter lesions may be predictors of neuropsychological deficits in post-stroke patients (Jokinen et al., 2005). AD patients with white matter changes at baseline show a rapid decline of cognitive function (de Leeuw et al., 2005).

* Corresponding authors. Chiu is to be contacted at Department of Neurology, National Taiwan University Hospital, No. 7, Chung-Shan S. Rd., Taipei, 100, Taiwan. Tel.: +886 968 661507; fax: +886 2 23418395. Chen, Department of Medical Imaging, National Taiwan University Hospital, No. 7, Chung-Shan S. Rd., Taipei, 100, Taiwan. Tel.: +8862 23123456x5634; fax: +886223324552.

E-mail addresses: avon@ha.mc.ntu.edu.tw (Y.-F. Chen), mjchiu@ntu.edu.tw (M.-J. Chiu).

1 Dr. C.C. Lin is an equal first author.
Frontotemporal dementia (FTD) is characterized by early language dysfunction or personality change. Two types of pathological features can be found. In some patients, there is microvacuolation of upper cortical layers, and in others, there is gliosis of cortical and subcortical white matter (Neary et al., 2005). The topography of the pathological changes corresponds to the cognitive domains involved, being restricted to frontal and temporal regions (Rosen et al., 2002). A recent diffusion tensor imaging study showed significant white matter damage in the early phase of FTD (Borroni et al., 2007).

Vascular dementia includes a heterogeneous group of patients presenting cognitive impairment from various cerebrovascular lesions. Impairments in cognitive function are related to the location and extent of the vascular insult (Gold et al., 2005). Subcortical ischemic vascular dementia (SIVD) is a subtype of vascular dementia with small lacunes in the basal ganglia, thalamus, or subcortical white matter regions. White matter disruption measured by diffusion tensor imaging was correlated with executive dysfunction in patients with SIVD (O'Sullivan et al., 2004).

Both AD and FTD have pathological features of cortical neuron loss and have been considered to be primary degenerative processes in the 
neocortex (Pearson et al., 1985; Rogers and Morrison, 1985). However, white matter involvement was invariably found in either group of patients. It could be secondary to the degeneration of cortical neurons or due to primary pathology occurring in the white matter regions. On the other hand, SIVD has primary white matter lesions from ischemic injury.

Current magnetic resonance imaging (MRI) technology has made possible the in vivo assessment of white matter integrity by using diffusion tensor imaging (DTI). In normal white matter tissue, axons are regularly aligned and well myelinated. Thus, the random diffusion of water molecules is restricted to a predominant diffusion orientation. Fractional anisotropy (FA) estimates this preference and is used to assess fiber tract integrity and alignment so that normal white matter tissue shows high FA (Pierpaoli et al., 1996). Mean diffusivity (MD) is a measure of the average motion of water molecules, which is independent of tissue directionality. Normal white matter tissue has low MD (Basser and Pierpaoli, 1996). Diseases that disrupt the directionality of fiber tracts and the tissue integrity of brain white matter reduce FA and increase MD (Englund et al., 2004).

A number of DTI studies have examined AD (Bozzali et al., 2002; Choi et al., 2005; Naggara et al., 2006), mild cognitive impairment (Fellgiebel et al., 2006; Medina et al., 2006) and cognitive declines associated with chronic vascular disease or leukoaraosis (O'Sullivan et al., 2004). There are few studies, however, on non-AD forms of dementia (Yoshiura et al., 2006). Furthermore, it is not feasible to carry out cross-study comparisons across different types of dementia because published studies have been performed with variable platforms using different methods of analysis such as region of interest (ROI), histogram, or voxel-by-voxel approaches (Bozzali and Cherubini, 2007). Thus, we investigated white matter integrity with DTI in patients with amnesic mild cognitive impairment (MCI), AD, vascular dementia (VaD), and frontotemporal dementia (FTD). The aim was to explore patterns specific or common to different types of dementia, and to understand more about the white matter pathology of dementia on an image platform with a uniform analysis method.

\section{Methods}

\subsection{Patients}

We examined 10 patients with MCI, 30 with AD, 18 with SIVD, 7 with FTD and 20 control subjects (Table 1 ). All subjects received a comprehensive clinical examination including medical history, physical and neurological examination, laboratory investigation and brain MRI study. Patients with MCI and various dementias were evaluated with the Mini-Mental State Examination, the Clinical Dementia Rating scale (CDR), the Behavioral Pathology in Alzheimer's Disease Rating Scale, Lawton's Instrumental Activities of Daily Living (IADL) and Barthel's Index for Activities of Daily Living (ADL). The CDR was used to evaluate all subjects who participated in the study except for the non-applicable FTD patients. CDR scores are interpreted as follows: $C D R=0$ indicates control subjects, $\mathrm{CDR}=0.5$ indicates $\mathrm{MCI}, \mathrm{CDR}=1$ indicates mild dementia, $\mathrm{CDR}=2$ indicates moderate dementia, and $\mathrm{CDR}=3$ indicates severe dementia.

Control subjects and subjects with $\mathrm{MCI}$ were assessed with the Wechsler Adult Intelligence Scale-III (WAIS-III) and the Taiwan version of the Wechsler Memory ScaleThird Edition (WMS-III; Hua et al., 2005). Subjects with deficits on any recall subtest of the WMS-III, a CDR score of 0.5 but normal ADL/IADL were diagnosed as MCI according to Mayo Clinic criteria (Petersen et al., 2001). Patients with AD fulfilled the NINCDS-ADRDA criteria for probable AD (McKhann et al., 1984). The diagnosis of vascular dementia was

\section{Table 1}

Demographic profile of the patients with various dementias and controls.

\begin{tabular}{llllll}
\hline & CS & MCI & AD & SIVD & FTD \\
\hline Number & 20 & 10 & 30 & 18 & 7 \\
Age yr (SD) & $70.1(7.1)$ & $71.0(9.1)$ & $73.8(10.7)$ & $78.2(87.7)$ & $61.0(7.6)^{*}$ \\
Gender (M/F) & $9 / 11$ & $7 / 3$ & $10 / 20$ & $7 / 11$ & $4 / 3$ \\
CDR $(1 / 2 / 3)$ & & & $18 / 11 / 1$ & $11 / 6 / 1$ &
\end{tabular}

(SD): standard deviation; (M/F): male versus female; CDR: clinical dementia rating; $C S$ : control subjects; $\mathrm{MCI}$ : amnesic mild cognitive impairment, AD: Alzheimer's disease; FTD: frontotemporal dementia; SIVD: subcortical ischemic vascular dementia; *ANOVA with Bonferroni post hoc test, patients with FTD significantly younger than patients with AD and SIVD (both $P<0.05$ ). made on the basis of NINDS-AIREN criteria excluding those patients with large vesse strokes such as major cerebral arteries, association cortical areas and watershed carotid territories (Roman et al., 1993; Van Straaten et al., 2003). Thus, we included only those patients with small vessel disease, such as multiple lacunae in the basal ganglia, thalamus and frontal white matter or patients with periventricular leukoaraosis. We studied patients with SIVD, which is a subtype of vascular dementia. The diagnosis of FTD was made on the basis of Neary's criteria (Neary et al., 1998). Control subjects were volunteers with normal cognitive function confirmed by the aforementioned neuropsychological test battery. All subjects were right-handed. All subjects or their principal caregivers gave their informed consent. The study was approved by the ethics committee of the university hospital.

\subsection{MRI acquisition}

Images were acquired with a 1.5-Tesla GE Excite MRI system. Routine whole brain MRI scans were available for every subject including axial T2-weighted image (TR/TE, 5600/90, slice thickness $5 \mathrm{~mm}$ ), axial fluid-attenuated inversion recovery (FLAIR) (TR/TI/TE 9000/ 2250/85, slice thickness $5 \mathrm{~mm}$ ), and coronal 3D T1WI using inversion prepared spoiled gradient echo (IR-SPGR) sequence (TR/TE/flip angle $10.5 / 2.2 / 15^{\circ}$, slice thickness $1.5 \mathrm{~mm}$ ). For DTI acquisition, we used single shot spin echo EPI acquisition with TR/TE $=6000 \mathrm{~ms} /$ $90 \mathrm{~ms}, 25$ uniformly distributed gradient directions, a $b$-value of $1000 \mathrm{~s} / \mathrm{mm}^{2}$, and a NEX of 2 . Twenty-four contiguous axial slices were acquired with 5 -mm slice thickness, $128 \times 128$ matrix, and $24 \mathrm{~cm} \times 24 \mathrm{~cm}$ field of view; the slices were positioned to run parallel to the anterior commissural and posterior commissural plane. The total acquisition time was $5 \mathrm{~min} 40 \mathrm{~s}$.

\subsection{DTI measurement and analysis}

Post-processing of the DTI data was done using a GE work station (FuncTool, Advantage Workstation 4.2, GE, Medical System, Milwaukee, WI). The system used automatic correction of the EPI distortion by scaling, de-skewing and translating to align each image with the reference image $(b=0)$ to minimize the mismatch between diffusion images and reference image. The MD and FA images were computed and displayed along with the reference image. We measured the MD and FA at different locations of white matter using ROI-based analysis. The rater, the neuroradiologist responsible for the placement of ROIs, was blind to the clinical diagnosis of all the participants. We placed 12 ovoid ROIs (size ranged from 30 to $40 \mathrm{~mm}^{2}$, containing 812 pixels) in the white matter regions on two slices that included the temporal horns on one slice, and the genu and splenium of the corpus callosum on the other slice (Figs. 1 and 2). The 12 selected regions included temporal lobe white matter adjacent to the temporal horns on either side, genu and splenium of the corpus callosum, anterior medial subcortical white matter, anterior periventricular white matter, posterior medial subcortical white matter and posterior periventricular white matter on the same slice of the genu and splenium of the corpus callosum. The non-midline regions were measured on either side. We selected the ROIs on images acquired without diffusion gradients $\left(b=0 \mathrm{~s} / \mathrm{mm}^{2}\right)$ and then transferred them onto both MD and FA images for each subject to avoid selection bias from different DTI parameters. Partial volume averaging from CSF space or gray matter structure was reduced by placing the ovoid sphere in the center of the target white matter and gradually adjusting the ROI size (812 pixels) to avoid the margin reaching cerebrospinal fluid space or gray matter. In case of subcortical white matter lacunae (equal to or smaller than $15 \mathrm{~mm}$ ), we placed the ROI in the adjacent white matter excluding the lacunar infarction lesion, while in those patients with periventricular leukoaraiosis, we placed the ROI as predefined. In case of atrophy of the corpus callosum, we aligned the long axis of the ovoid sphere along the direction of the fiber tract and made a slender and small ROI, e.g. $2 \times 4$ pixels, to avoid partial volume effected. This was the advantage of using ovoid sphere ROIs of variable size.

\subsection{Statistical analysis}

Demographic and clinical data with non-parametric characteristics were compared by Chi-square test, and those with parametric characteristics were analyzed with analysis of variance (ANOVA) with the Bonferroni post hoc test.

Levene's test was performed to determine whether the variances between the patient group and their age-matched controls were equal. Both variances of FA and MD violated the homogeneity test; thus, the Mann-Whitney $U$ test was used for betweengroup comparisons of MD and FA in every ROI.

Spearman correlation analysis was used to assess the relation between DTI and clinical severity measured by global CDR scores. All tests of statistic significance were two-tailed. Statistics were performed with SPSS software package version 11.0 (SPSS, Chicago, IL, 2003).

\section{Results}

There was a significant group effect of age $(F=5.4, P<0.01)$. A post hoc test showed that patients with FTD were younger than patients with AD and SIVD (both $P<0.05$ ). However, the pairwise post hoc test showed no significant age effect between control subjects and patients with $\mathrm{MCI}$ or other dementias (all $P>0.05$ ). There was no gender effect between groups 


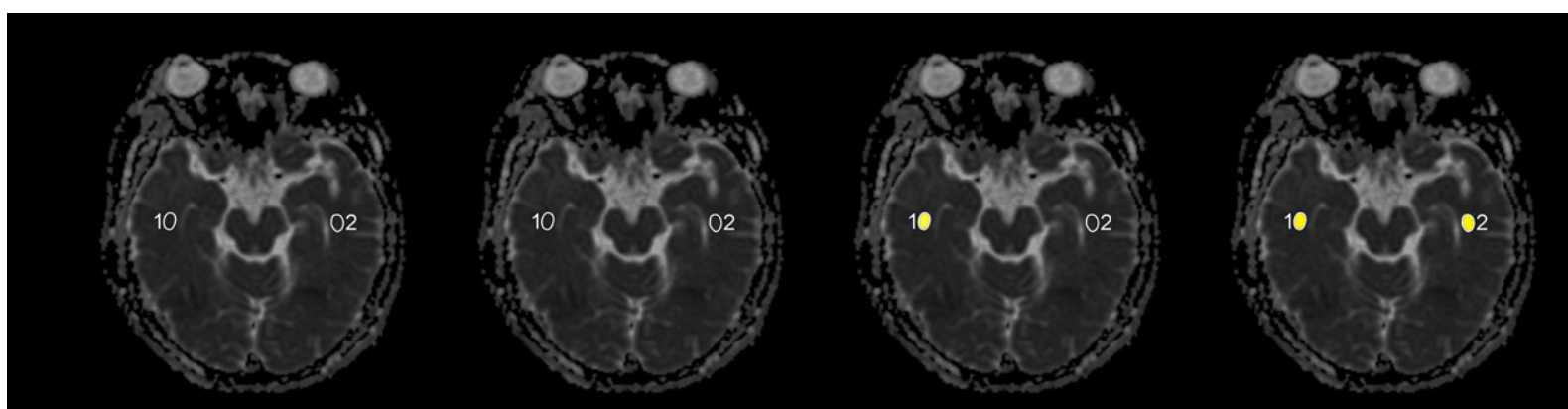

MD

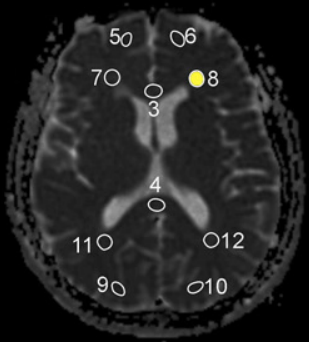

$\mathrm{R}$

$\mathrm{MCl}$

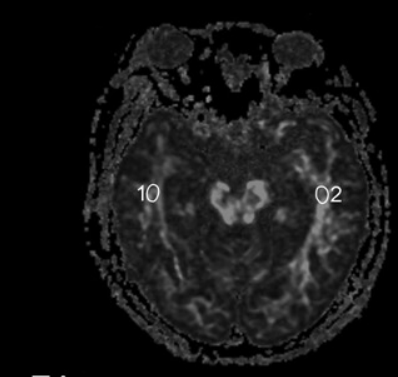

FA

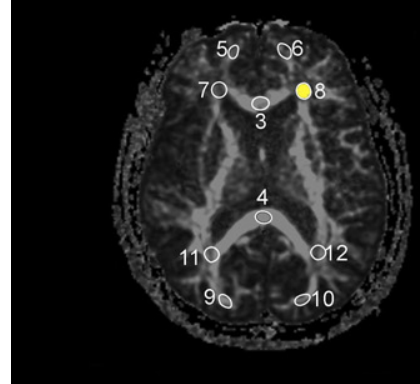

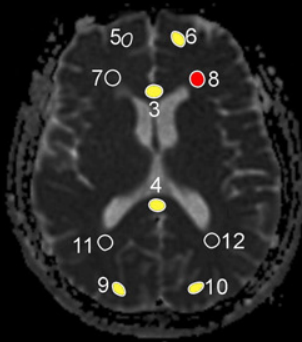

$A D$
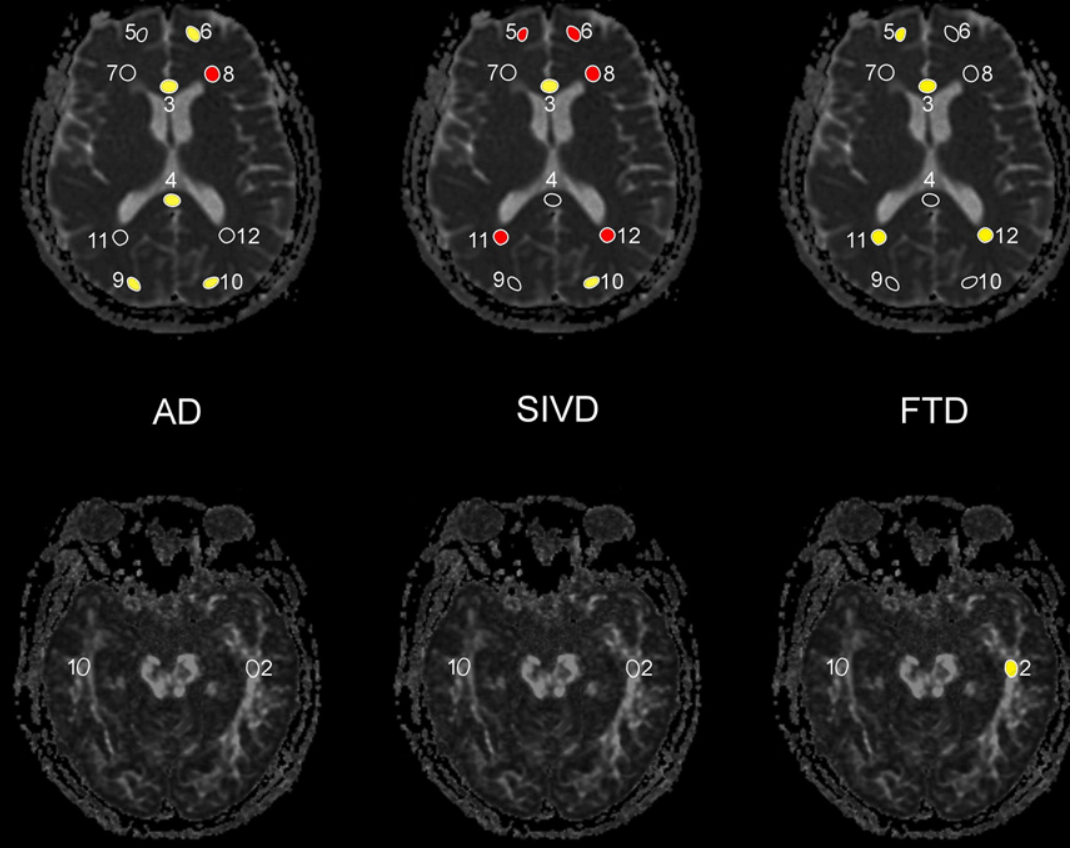

FTD

$L$

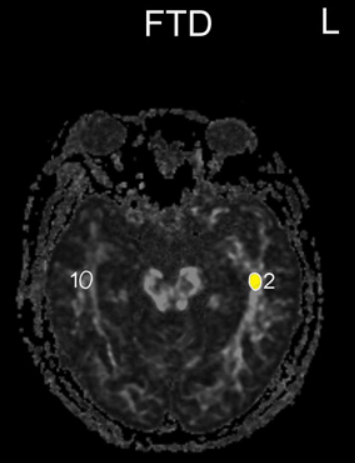

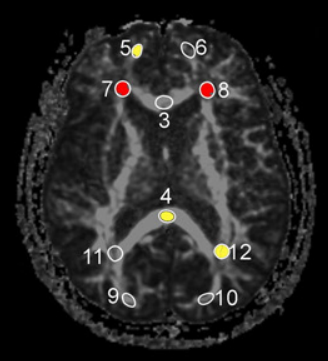

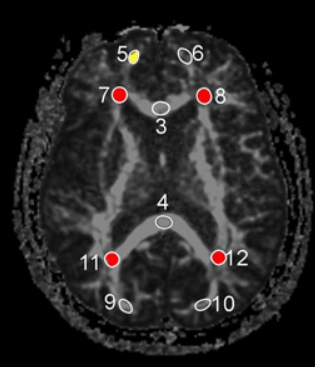

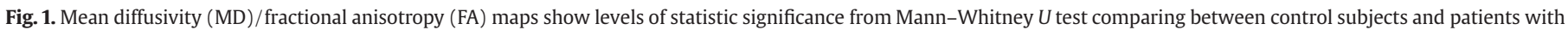

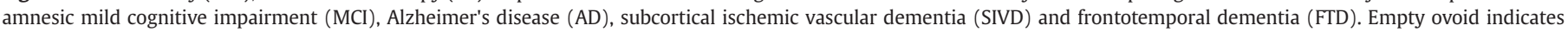

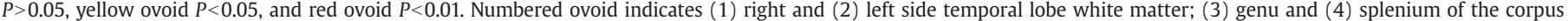

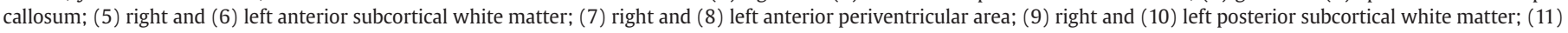
right and (12) left posterior periventricular area. $\mathrm{R}$ indicates right side and $\mathrm{L}$ for left side.

(Pearson Chi-square $=4.82, P>0.1$ ). The severity of dementia was about equivalent $\left(\chi^{2}=0.171, P>0.5\right)$ as assessed by the Clinical Dementia Rating (CDR; Hughes et al., 1982) in patients with AD (CDR 1/2/3: 18/11/1) and patients with SIVD (CDR 1/2/3:11/6/1; Table 1$)$.

Compared with controls, patients with $\mathrm{MCI}$ had increased $\mathrm{MD}(Z=$ $-2.42, P<0.05)$ and reduced FA $(Z=-2.09, P<0.05)$ in the left anterior periventricular area. Additional areas showing trends toward significant differences were the right posterior periventricular area $(Z=-1.89, P=0.058$ for $\mathrm{MD} ; Z=-1.80, P=0.71$ for $\mathrm{FA})$ and the genu of the corpus callosum ( $Z=-1.76, P=0.078$ for FA). Compared with the controls, $\mathrm{AD}$ patients showed increased MD in the genu $(Z=$
$-2.27, P<0.05)$ and splenium $(Z=-2.49, P<0.05)$ of the corpus callosum, left anterior subcortical $(Z=-2.35, P<0.05)$ and bilateral posterior subcortical white matter $(Z=-2.03, P<0.05$ on the left; $Z=-2.05, P<0.05$ on the right side), and the left anterior periventricular area $(Z=-2.94, P<0.01)$. AD patients had reduced $F A$ in the splenium of the corpus callosum $(Z=-2.01, P<0.05)$, bilateral anterior periventricular areas $(Z=-3.57$ on the left and $Z=-3.40$ on the right side, both $P<0.01$ ), left posterior periventricular area $(Z=-2.20, P<0.05)$ and right anterior subcortical area $(Z=-2.35$, $P<0.05)$. SIVD patients showed a diffuse increase of MD in multiple areas, including the bilateral anterior subcortical areas $(Z=-3.38$ on 


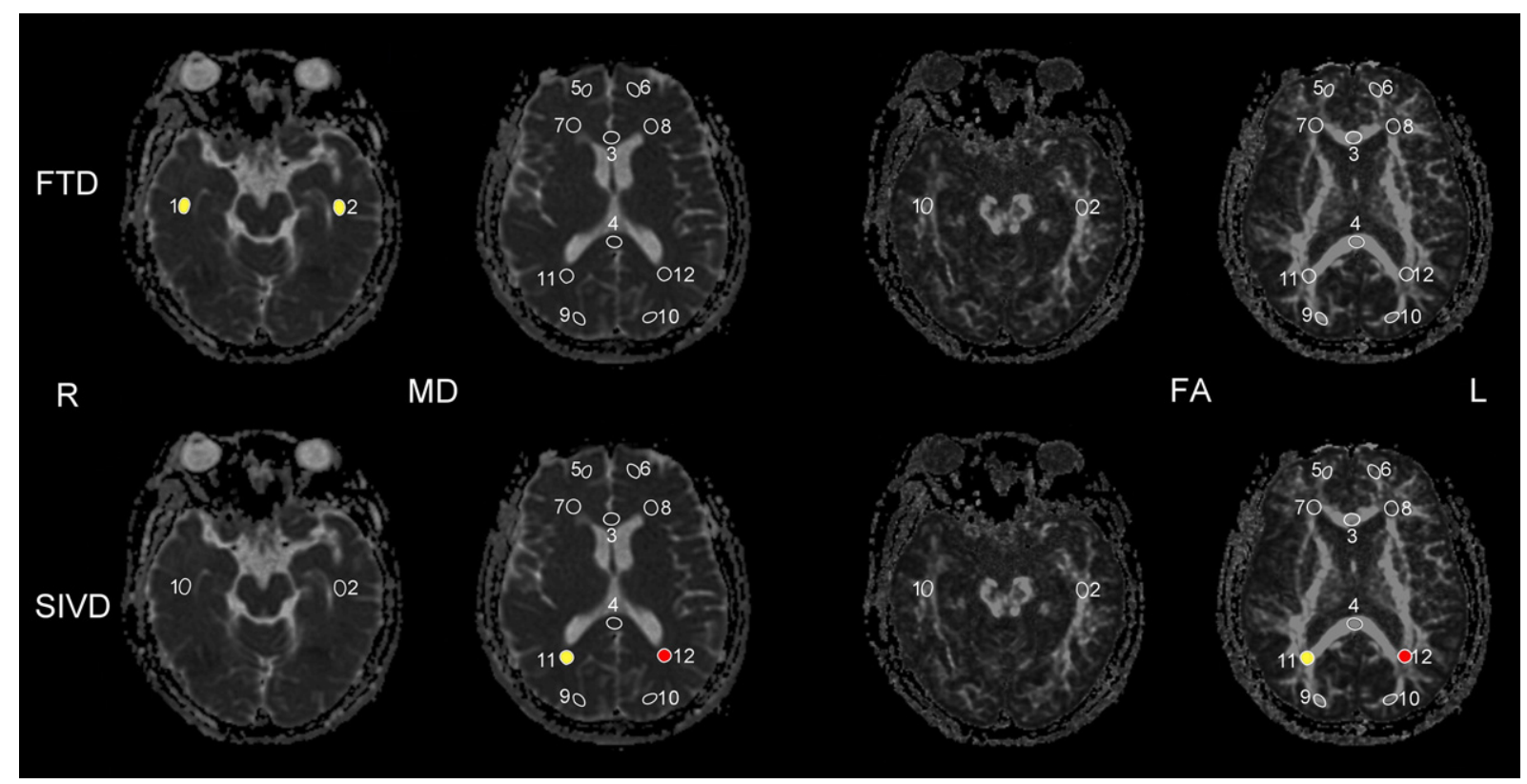

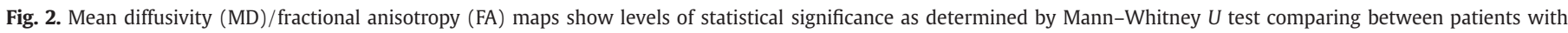

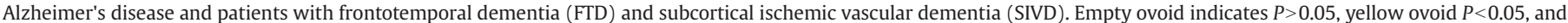

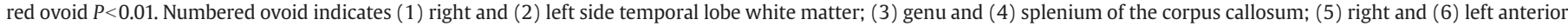

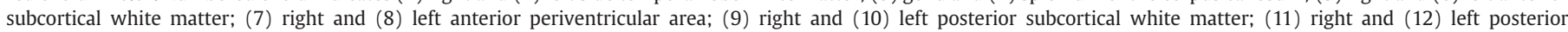
periventricular area. $\mathrm{R}$ indicates right side and $\mathrm{L}$ for left side.

the left and $Z=-2.95$, on the right side, both $P<0.01$ ) and left posterior subcortical area $(Z=-2.46, P<0.05)$, left anterior periventricular $(Z=-3.73, P<0.001)$ and bilateral posterior periventricular areas $(Z=-3.30$ on the left and $Z=-2.94$, on the right side, both $P<0.01)$, and the genu of the corpus callosum $(Z=-2.00, P<0.05)$. SIVD patients had a significant reduction of FA in the periventricular areas of the bilateral anterior $(Z=-3.85, P<0.001$ on the left and $Z=$ $-3.10, P<0.01$ on the right side) and bilateral posterior aspects $(Z=$ $-4.42, P<0.001$ on the left, $Z=-3.09, P<0.01$ on the right), and also in the right anterior subcortical area $(Z=-2.11, P<0.05)$. FTD patients had increased MD in the bilateral temporal areas $(Z=-2.38$ on the left and $Z=-2.16$ on the right side, both $P<0.05$ ), the genu of the corpus callosum $(Z=-1.99, P<0.05)$, the right anterior subcortical area $(Z=-2.05, P<0.05)$, and the bilateral posterior periventricular areas $(Z=-2.49$ on the left and $Z=-2.33$ on the right side, both $P<0.05)$. Reduction of FA was also observed in FTD patients in the left temporal area $(Z=-2.02, P<0.043)$ and the right anterior periventricular areas $(Z=-2.99, P<0.01)$. Results were summarized in Tables 2 and 3, and illustrated in Fig. 1.

We also compared $\mathrm{MCI}$ with the various types of dementia (Fig. 2). Taking AD as a prototype of dementia, we compared MCI, SIVD and FTD with $\mathrm{AD}$. There was no significant difference between $\mathrm{MCl}$ and $\mathrm{AD}$ in all ROIs with either MD or FA measurements. Patients with SIVD had higher MD in bilateral posterior periventricular areas $(Z=-2.64, P<0.01$ on the left and $Z=-2.18, P<0.05$ on the right side) and lower FA in bilateral posterior periventricular areas $(Z=-2.66, P<0.01$ on the left and $Z=-2.39, P<0.05$ on the right side) than AD patients had. FTD patients had higher MD in bilateral temporal areas $(Z=-2.50$ on the left and $Z=-2.06$ on the right side, both $P<0.05$; Fig. 2) than $A D$ patients had.

A correlation analysis of the MD and FA of each ROI with clinical severity measured by global CDR scores was performed in controls $(\mathrm{CDR}=0)$, patients with $\mathrm{MCI}(\mathrm{CDR}=0.5)$, and $\mathrm{AD}(\mathrm{CDR}=1,2$ or 3$)$ and $\mathrm{VD}(\mathrm{CDR}=1,2$ or 3$)$. MD was positively associated with the global

Table 2

Mean diffusivity of ROI in control, $\mathrm{MCI}$ and various dementias.

\begin{tabular}{|c|c|c|c|c|c|c|c|}
\hline $\operatorname{MD}\left(10^{-9} \mathrm{~m}^{2} / \mathrm{s}\right)$ & Temporal & Ant SC & Ant PV & Post SC & Post PV & \multicolumn{2}{|c|}{ Corpus callosum } \\
\hline \multicolumn{8}{|l|}{ CS (20) } \\
\hline Right & $0.86(0.07)$ & $0.76(0.03)$ & $0.88(0.09)$ & $0.81(0.04)$ & $0.85(0.04)$ & Genu & $0.87(0.06)$ \\
\hline Left & $0.87(0.07)$ & $0.69(0.05)$ & $0.78(0.05)$ & $0.82(0.05)$ & $0.87(0.10)$ & Splenium & $0.82(0.21)$ \\
\hline \multicolumn{8}{|l|}{$\mathrm{MCI}(10)$} \\
\hline Right & $0.88(0.07)$ & $0.76(0.06)$ & $0.87(0.09)$ & $0.79(0.09)$ & $0.93(0.12)$ & Genu & $0.88(0.06)$ \\
\hline Left & $0.87(0.06)$ & $0.70(0.05)$ & $0.85(0.07)^{*}$ & $0.79(0.08)$ & $0.92(0.13)$ & Splenium & $0.98(0.20)$ \\
\hline \multicolumn{8}{|l|}{$\mathrm{AD}(30)$} \\
\hline Right & $0.89(0.07)$ & $0.79(0.11)$ & $0.95(0.19)$ & $0.78(0.07)^{*}$ & $0.92(0.17)$ & Genu & $0.95(0.16)^{*}$ \\
\hline Left & $0.86(0.07)$ & $0.74(0.10)^{*}$ & $0.93(0.24)^{* *}$ & $0.78(0.06)^{*}$ & $0.91(0.16)$ & Splenium & $0.98(0.20)^{*}$ \\
\hline \multicolumn{8}{|l|}{ FTD (7) } \\
\hline Right & $0.96(0.11) * \dagger$ & $0.86(0.19)^{*}$ & $1.14(0.35)$ & $0.81(0.09)$ & $0.99(0.15)^{*}$ & Genu & $1.11(0.43)^{*}$ \\
\hline Left & $1.03(0.17)^{* \dagger}$ & $0.83(0.30)$ & $1.09(0.39)$ & $0.76(0.10)$ & $0.99(0.13)^{*}$ & Splenium & $0.95(0.14)$ \\
\hline \multicolumn{8}{|l|}{$\operatorname{SIVD}(18)$} \\
\hline Right & $0.92(0.10)^{*}$ & $0.82(0.08)^{* *}$ & $0.96(0.16)$ & $0.82(0.10)$ & $1.06(0.26)^{* * \dagger}$ & Genu & $0.95(0.12) *$ \\
\hline Left & $0.92(0.12)$ & $0.79(0.09)^{* *}$ & $0.99(0.21)^{* *}$ & $0.77(0.08)^{*}$ & $1.08(0.23)^{* * t}$ & Splenium & $0.97(0.23)$ \\
\hline
\end{tabular}

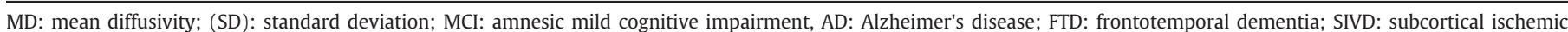

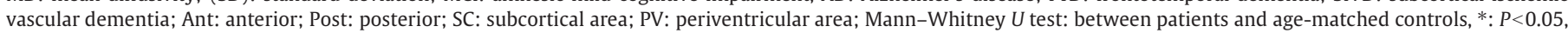
**: $P<0.01$, ***: $P<0.001$; between AD patients and other dementias, $\dagger: P<0.05, \ddagger: P<0.01$. 
Table 3

Fractional anisotropy of ROI in control, $\mathrm{MCI}$ and various dementias.

\begin{tabular}{|c|c|c|c|c|c|c|c|}
\hline $\mathrm{FA}$ & Temporal & Ant SC & Ant PV & Post SC & Post PV & \multicolumn{2}{|c|}{ Corpus callosum } \\
\hline \multicolumn{8}{|l|}{ CS (20) } \\
\hline Right & $0.45(0.06)$ & $0.45(0.04)$ & $0.37(0.07)$ & $0.44(0.06)$ & $0.48(0.07)$ & Genu & $0.74(0.05)$ \\
\hline Left & $0.48(0.05)$ & $0.46(0.06)$ & $0.39(0.06)$ & $0.44(0.04)$ & $0.49(0.07)$ & Splenium & $0.78(0.06)$ \\
\hline \multicolumn{8}{|l|}{$\mathrm{MCI}(10)$} \\
\hline Right & $0.45(0.06)$ & $0.43(0.05)$ & $0.34(0.10)$ & $0.45(0.05)$ & $0.45(0.09)$ & Genu & $0.77(0.02)$ \\
\hline Left & $0.47(0.06)$ & $0.45(0.04)$ & $0.34(0.08)^{*}$ & $0.46(0.07)$ & $0.44(0.09)$ & Splenium & $0.74(0.07)$ \\
\hline \multicolumn{8}{|l|}{$\mathrm{AD}(30)$} \\
\hline Right & $0.43(0.05)$ & $0.42(0.06)$ & $0.30(0.08)^{* *}$ & $0.43(0.07)$ & $0.44(0.09)$ & Genu & $0.74(0.08)$ \\
\hline Left & $0.46(0.05)$ & $0.45(0.06)^{*}$ & $0.31(0.10)^{* *}$ & $0.44(0.06)$ & $0.43(0.09)^{*}$ & Splenium & $0.73(0.09)^{*}$ \\
\hline \multicolumn{8}{|l|}{ FTD (7) } \\
\hline Right & $0.44(0.07)$ & $0.39(0.10)$ & $0.24(0.09)^{*}$ & $0.48(0.09)$ & $0.38(0.09)^{*}$ & Genu & $0.65(0.20)$ \\
\hline Left & $0.42(0.06)^{*}$ & $0.42(0.12)$ & $0.31(0.12)$ & $0.46(0.08)$ & $0.42(0.08)^{*}$ & Splenium & $0.75(0.07)$ \\
\hline \multicolumn{8}{|l|}{ SIVD (18) } \\
\hline Right & $0.41(0.06)$ & $0.41(0.12)^{*}$ & $0.29(0.12)^{* *}$ & $0.46(0.10)$ & $0.37(0.10)^{* *} \dagger$ & Genu & $0.73(0.07)$ \\
\hline Left & $0.45(0.06)$ & $0.44(0.10)$ & $0.29(0.10)^{* *}$ & $0.46(0.06)$ & $0.35(0.09)^{* *} \ddagger$ & Splenium & $0.74(0.14)$ \\
\hline
\end{tabular}

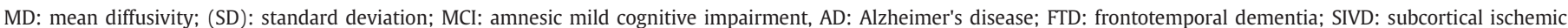

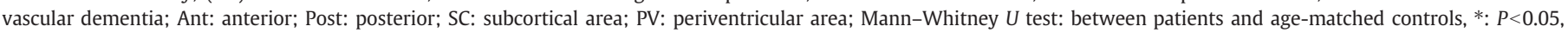
**: $P<0.01$; between $\mathrm{AD}$ patients and other dementias, $\dagger: P<0.05$, $\ddagger$ : $P<0.01$.

CDR score in the majority of ROIs, while FA was negatively associated with the global CDR score mainly in the anterior and posterior periventricular areas (Table 4, Fig. 3).

\section{Discussion}

Previous DTI studies demonstrated associations of pathology in brain white matter with progression of cognitive deficits (de Leeuw et al., 2005; Fellgiebel et al., 2006; Yoshiura et al., 2002). Our study using DTI showed patterns of white matter abnormalities in patients with different types of dementia.

$\mathrm{AD}$ patients had diffusion tensor changes in both the anterior and posterior cerebral white matter, including the genu and the splenium of the corpus callosum. The splenium of the corpus callosum was the area specific to AD (Fellgiebel et al., 2005; Yamauchi et al., 2000). The observation of predominantlyy anterior involvement is compatible with previous studies measuring brain diffusivity (Hanyu et al., 1998, 1999; Choi et al., 2005; Naggara et al., 2006; Sjöbeck et al., 2006; Duan et al., 2006; Zhang et al., 2007), and with studies assessing T2-weighted lesions of the brain MRI (Brun and Englund, 1986; Bracco et al., 2005). Early changes in frontal white matter may be an indicator of a later diagnosis of AD, in line with the retro-genesis theory (Choi et al., 2005; Naggara et al., 2006). The idea is further supported by the fact that our $\mathrm{MCI}$ patients showed diffusion tensor change only in the left anterior periventricular area and a trend toward a change in the genu of the corpus callosum. The findings are in line with the designation of $\mathrm{MCl}$ as prodromal stage of AD (Perry and Hodges, 1999; Geslani et al., 2005). However, DTI white matter change has also been reported in the temporal lobes and in posterior areas in AD patients (Duan et al., 2006; Medina et al., 2006; Naggara et al., 2006). We also found diffusion tensor change in anterior (frontal) and posterior (parieto-occipital) subcortical areas. Thus, the issue of the anterior or posterior predominance in $\mathrm{AD}$ remains to be settled in terms of diffusion tensor change.

FTD patients showed white matter change in the bilateral temporal, anterior (frontal) subcortical, anterior and posterior periventricular areas and in the genu of the corpus callosum. It was proposed that observed white matter abnormalities may be secondary to damage in the overlying cortex (Yoshiura et al., 2006), which may account for frontal and temporal diffusion tensor changes while the anterior periventricular areas mostly convey the frontal related fiber tracts. The findings are compatible with the concept of a focal dementia with regional brain atrophy typically in the frontal and temporal areas (Boccardi et al., 2003).

The genu of the corpus callosum was involved both in FTD and AD. The genu of the corpus callosum is an important myelinated axonal relay connecting cortical-cortical and cortical-subcortical areas between two cerebral hemispheres. In this study the splenium of the corpus callosum was involved only in the $\mathrm{AD}$ group, compatible with a previous report (Fellgiebel et al., 2005). The pattern of corpus callosum involvement is in agreement with a previous study indicating that atrophy in FTD mainly occurred in the anterior quarter, whereas atrophy in AD mainly occurred in the posterior quarter (Yamauchi et al., 2000).

SIVD patients showed diffusion tensor changes in anterior and posterior periventricular areas, bilateral anterior (frontal) subcortical areas, and the genu of the corpus callosum as compared with the control subjects. The bilateral diffusion tensor change in the frontal subcortical areas is specific for SIVD, which may illustrate the clinical and neuropsychological features characterized by executive deficit or subcortical mental slowness. Extensive periventricular diffusion tensor changes also characterized SIVD patients. Our SIVD patients had periventricular leukoaraiosis or subcortical lacunar infarcts. White matter diffusivity was reported to be associated with full scale IQ and with scores on executive function tests in previous studies (O'Sullivan et al., 2004).

Correlation analysis revealed that MD increased extensively with the progression of the clinical severity of dementia. However, FA correlated with clinical severity mostly in the anterior and posterior periventricular areas. On the one hand, this correlation confirms the importance of periventricular areas in cognitive function, and on the other hand, it suggests a differential sensitivity between FA and MD. FA is more sensitive in areas with good fiber directionality such as in the periventricular areas where long-projecting fibers of the cholinergic pathways trail along. Nevertheless, a recent study using postmortem brain to probe pathology underlying diffusion tensor change found that both MD and FA were highly correlated with myelin content and axon count (Schmierer et al., 2008).

Table 4

Correlation coefficients between MD, FA and CDR.

\begin{tabular}{lcclll}
\hline & MD & & & FA & \\
\cline { 2 - 3 } \cline { 5 - 6 } & Right & Left & & Right & Left \\
\hline Temporal & $0.343^{* *}$ & 0.189 & & -0.194 & -0.064 \\
Ant SC & $0.293^{* *}$ & $0.419^{* *}$ & & $-0.324^{* *}$ & -0.129 \\
Ant PV & $0.243^{*}$ & $0.444^{* *}$ & & $-0.439^{* *}$ & $-0.523^{* *}$ \\
Post SC & -0.007 & 0.174 & & -0.141 & -0.005 \\
Post PV & $0.298^{* *}$ & $-0.243^{*}$ & & $-0.308^{* *}$ & $-0.439^{* *}$ \\
Genu & $0.314^{* *}$ & & & -0.079 & \\
Splenium & $0.241^{*}$ & & & -0.203 &
\end{tabular}

MD: mean diffusivity; FA: fractional anisotropy; CDR: clinical dementia rating; Ant: anterior; Post: posterior; SC: subcortical area; PV: periventricular area; Correlation coefficient (Spearman's rho), ${ }^{*}: P<0.05$, ${ }^{* *}: P<0.01$ in controls, patients with mild cognitive impairment, Alzheimer's disease and subcortical ischemic vascular dementia. 


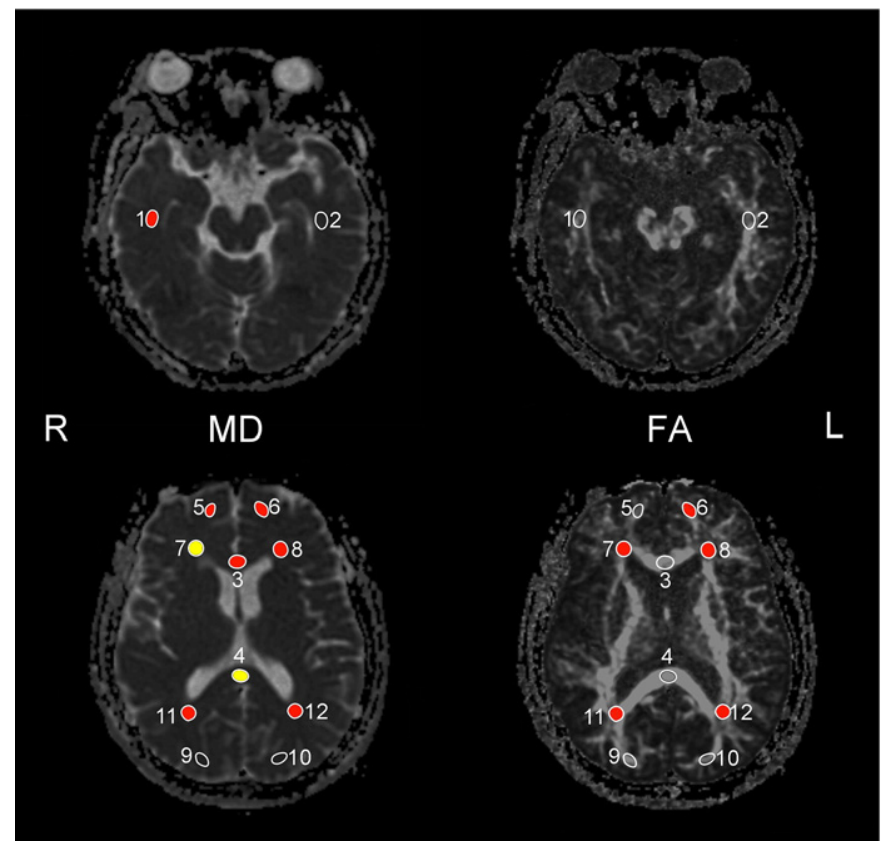

Fig. 3. Mean diffusivity (MD)/fractional anisotropy (FA) maps show levels of statistical significance from Spearman's correlation between MD/FA measurements and scores of clinical dementia ratings of control subjects, patients with amnesic mild cognitive impairment, Alzheimer's disease, and subcortical ischemic vascular dementia. Empty ovoid indicates $P>0.05$, yellow ovoid for $P<0.05$, and red ovoid $P<0.01$. Numbered ovoid indicates (1) right and (2) left side temporal lobe white matter; (3) genu and (4) splenium of the corpus callosum; (5) right and (6) left anterior subcortical white matter; (7) right and (8) left anterior periventricular area; (9) right and (10) left posterior subcortical white matter; (11) right and (12) left posterior periventricular area. $\mathrm{R}$ indicates right side and $\mathrm{L}$ for left side.

The lateral part of the cholinergic pathway originates from the nucleus of Meynert located in the basal forebrain, and the trajectories project to the frontal, temporal, parietal and occipital cortices alongside the lateral ventricles (Selden et al., 1998). The cholinergic modulation is considered critical for attention and memory (Mesulam, 2004). The severity of periventricular hyperintensities was associated with the severity of dementia (de Groot et al., 2000) and was related to poor efficacy of cholinesterase inhibitors in patients with AD (Fukui et al., 2006). The loss of the cholinergic pathways was also reported in patients with vascular dementia (Tomimoto et al., 2005).

There are some limitations of the present study. We used anisotropic voxels in this DTI study, which might cause a bias in the calculation of FA. Although it is not unusual that non-isotropic voxels are used for DTI measurement in the clinical setting as a trade-off between throughplane resolution and scanning time. Oouchi et al. (2007) compared isotropic with non-isotropic diffusion anisotropy measurement. They found possible underestimation of FA in areas with crossing fibers such as the superior longitudinal fasciculus, thalamus, and red nucleus but not in the corpus callosum, posterior limb of the internal capsule, and cortical spinal tract at the semiovale level. The underestimation was not found with MD. However, this is a minor point since we compared DTI measurement between groups. The other limitation of the study was inherent in the ROI-based analysis. The anatomic sites chosen for ROI measurements may not be comprehensive enough to study the diseaserelated white matter change. Generally speaking, ROI-based analysis is time-consuming and operator-dependent. Artifacts are related to the shape and size of the ROIs used. The use of small-sized ROIs reduces partial volume effects and the use of large-sized ROIs reduces noise (Bozzali and Cherubini, 2007). In this study, we had experienced hands for placing ROIs who followed strict criteria for selecting ROIs which substantiated a good intra-observer (intra-class correlation [ICC] 0.97 for MD and 0.98 for FA) and inter-observer reliability (ICC 0.97 for MD and 0.92 for $\mathrm{FA}$ ). We used variable-sized ROIs (30 to $40 \mathrm{~mm}^{2}$ ) to optimize the trade-off of minimal partial volume effect and low noise (Huang et al., 2007; Bozzali and Cherubini, 2007).

In conclusion, we demonstrated patterns of white matter change specific to $\mathrm{MCI}$ and various dementias through measuring diffusivity and anisotropy in multiple brain areas. $\mathrm{MCl}$ showed effects in the anterior periventricular area and possibly, in the genu of the corpus callosum; AD showed white matter change in both frontal and parieto-occipital subcortical areas, and in the splenium of the corpus callosum; SIVD showed white matter change in bilateral periventricular and frontal subcortical areas; and FTD revealed the typical bilateral temporal and frontal subcortical white matter change. Locations of white matter change common to various dementias were identified in the periventricular area, which implies a critical role of the lateral cholinergic pathways; and also in the genus of the corpus callosum, which is an important relay connecting cortical-cortical and cortical-subcortical areas between two hemispheres. Our study provides potential implications of DTI in the differential diagnosis of patients with different forms of dementia.

\section{Acknowledgement}

The study was supported in part by a grant from National Taiwan University Hospital (NTUH 93A16-3) and in part by a grant from the Taiwan National Science Council (NSC 95-2314-B-144).

\section{References}

Arnold, S.E., Hyman, B.T., Flory, J., Damasio, A.R., Van Hoesen, G.W., 1991. The topographical and neuroanatomical distribution of neurofibrillary tangles and neurititc plaques in the cerebral cortex of patients with Alzheimer's disease. Cerebral Cortex 1, 103-116.

Basser, P.J., Pierpaoli, C., 1996. Microstructural and physiological features of tissues elucidated by quantitative-diffusion-tensor MRI. Journal of Magnetic Resonance 111, 209-219.

Boccardi, M., Laakso, M.P., Bresciani, L., Galluzzi, S., Geroldi, C., Beltramello, A., Soininen, H., Frisoni, G.B., 2003. The MRI pattern of frontal and temporal brain atrophy in fronto-temporal dementia. Neurobiology of Aging 24, 95-103.

Borroni, B., Brambati, S.M., Agosti, C., Gipponi, S., Bellelli, G., Gasparotti, R., Garibotto, V., Di, L.M., Scifo, P., Perani, D., Padovani, A., 2007. Evidence of white matter changes on diffusion tensor imaging in frontotemporal dementia. Archives of Neurology 64 246-251.

Bozzali, M., Cherubini, A., 2007. Diffusion tensor MRI to investigate dementias: a brief review. Magnetic Resonance Imaging 25, 969-977.

Bozzali, M., Falini, A., Franceschi, M., Cercignani, M., Zuffi, M., Scotti, G., Comi, G., Filippi M., 2002. White matter damage in Alzheimer's disease assessed in vivo using diffusion tensor magnetic resonance imaging. Journal of Neurology, Neurosurgery \& Psychiarty 72, 742-746.

Bracco, L., Piccini, C., Moretti, M., Mascalchi, M., Sforza, A., Nacmias, B., Cellini, E. Bagnoli, S., Sorbi, S., 2005. Alzheimer's disease: role of size and location of white matter changes in determining cognitive deficits. Dementia and Geriatric Cognitive Disorders 20, 358-366.

Brun, A., Englund, E., 1986. A white matter disorder in dementia of the Alzheimer type: a pathoanatomical study. Annals of Neurology 19, 253-262.

Choi, S.J., Lim, K.O., Monteiro, I., Reisberg, B., 2005. Diffusion tensor imaging of frontal white matter microstructure in early Alzheimer's disease: a preliminary study. Journal of Geriatric Psychiatry and Neurology 18, 12-19.

de Groot, J.C., de Leeuw, F.E., Oudkerk, M., Van Gijn, J., Hofman, A., Jolles, J., Breteler, M.M. 2000. Cerebral white matter lesions and cognitive function: the Rotterdam Scan Study. Annals of Neurology 47, 145-151.

de Leeuw, F.E., Barkhof, F., Scheltens, P., 2005. Progression of cerebral white matter lesions in Alzheimer's disease: a new window for therapy? Journal of Neurology, Neurosurgery \& Psychiarty 76, 1286-1288.

Duan, J.H., Wang, H.Q., Xu, J., Lin, X., Chen, S.Q., Kang, Z., Yao, Z.B., 2006. White matter damage of patients with Alzheimer's disease correlated with the decreased cognitive function. Surgical and Radiologic Anatomy 28, 150-156.

Englund, E., Sjöbeck, M., Brockstedt, S., Lätt, J., Larsson, E.M., 2004. Diffusion tensor MRI post mortem demonstrated cerebral white matter pathology. Journal of Neurology 251, 350-352.

Fellgiebel, A., Müller, M.J., Wille, P., Dellani, P.R., Scheurich, A., Schmidt, L.G., Stoeter, P., 2005. Color-coded diffusion-tensor-imaging of posterior cingulate fiber tracts in mild cognitive impairment. Neurobiology of Aging 26, 1193-1198.

Fellgiebel, A., Dellani, P.R., Greverus, D., Scheurich, A., Stoeter, P., Müller, M.J., 2006 Predicting conversion to dementia in mild cognitive impairment by volumetric and diffusivity measurements of the hippocampus. Psychiatry Research 146, 283-287.

Fukui, T., Hieda, S., Bocti, C., 2006. Do lesions involving the cortical cholinergic pathways help or hinder efficacy of donepezil in patients with Alzheimer's disease? Dementia and Geriatric Cognitive Disorders 22, 421-431. 
Garde, E., Lykke, M.E., Rostrup, E., Paulson, O.B., 2005. Decline in intelligence is associated with progression in white matter hyperintensity volume. Journal of Neurology, Neurosurgery \& Psychiarty 76, 1289-1291.

Geslani, D.M., Tierney, M.C., Herrmann, N., Szalai, J.P., 2005. Mild cognitive impairment: an operational definition and its conversion rate to Alzheimer's disease. Dementia and Geriatric Cognitive Disorders 19, 383-389.

Gold, G., Kövari, E., Herrmann, F.R., Canuto, A., Hof, P.R., Michel, J.P., Bouras, C., 2005. Giannakopoulos P. Cognitive consequences of thalamic, basal ganglia, and deep white matter lacunes in brain aging and dementia. Stroke 36, 1184-1188.

Hanyu, H., Imon, Y., Sakurai, H., Iwamoto, T., Takasaki, M., Shindo, H., 1998. Diffusionweighted magnetic resonance and magnetization transfer imaging in the assessment of ischemic human stroke. Internal Medicine 37, 360-365.

Hanyu, H., Asano, T., Sakurai, H., Imon, Y., Iwamoto, T., Takasaki, M., Shindo, H., 1999 Diffusion-weighted and magnetization transfer imaging of the corpus callosum in Alzheimer's disease. Journal of Neurological Science 167, 37-44.

Hua, M.S., Chang, B.S., Lin, K.N., Yang, J.M., Lu, S.R., Chen, S.Y., 2005. Wechsler memory scale, 3rd ed. Chinese Behavioral Science Corporation, Taipei.

Huang, J., Friedland, R.P., Auchus, A.P., 2007. Diffusion tensor imaging of normalappearing white matter in mild cognitive impairment and early Alzheimer disease: preliminary evidence of axonal degeneration in the temporal lobe. American Journal of Neuroradiology 28, 1934-1948.

Hughes, C.P., Berg, L., Danziger, W.L., Coben, L.A., Martin, R.L., 1982. A new clinical scale for the staging of dementia. British Journal of Psychiatry 140, 566-572.

Jokinen, H., Kalska, H., Mäntylä, R., Ylikoski, R., Hietanen, M., Pohjasvaara, T., Kaste, M. Erkinjuntti, T., 2005. White matter hyperintensities as a predictor of neuropsychological deficits post-stroke. Journal of Neurology, Neurosurgery \& Psychiarty 76, 1229-1233.

McKhann, G., Drachman, D., Folstein, M., Katzman, R., Price, D., Stadlan, E.M., 1984 Clinical diagnosis of Alzheimer's disease: report of the NINCDS-ADRDA Work Group under the auspices of Department of Health and Human Services Task Force on Alzheimer's Disease. Neurology 34, 939-944.

Medina, D., de Toledo-Morrell, L., Urresta, F., Gabrieli, J.D., Moseley, M., Fleischman, D., Bennett, D.A., Leurgans, S., Turner, D.A., Stebbins, G.T., 2006. White matter changes in mild cognitive impairment and AD: a diffusion tensor imaging study. Neurobiology of Aging 27, 663-672.

Mesulam, M.M., 2004. The cholinergic innervation of the human cerebral cortex. Progress in Brain Research 145, 67-78.

Naggara, O., Oppenheim, C., Rieu, D., Raoux, N., Rodrigo, S., Dalla Barba, G., Meder, J.F., 2006 Diffusion tensor imaging in early Alzheimer's disease. Psychiatry Research 146, 243-249.

Neary, D., Snowden, J.S., Gustafson, L., Passant, U., Stuss, D., Black, S., Freedman, M., Kertesz, A., Robert, P.H., Albert, M., Boone, K., Miller, B.L., Cummings, J., Benson, D.F., 1998. Frontotemporal lobar degeneration: a consensus on clinical diagnostic criteria. Neurology 51, 1546-1554.

Neary, D., Snowden, J., Mann, D., 2005. Frontotemporal dementia. Lancet Neurology 4 , 771-780.

Oouchi, M., Yamada, K., Sakai, K., Kubota, T., Ito, H., Nishimura, T., 2007. Diffusion anisotropy measurement of brain white matter is affected by voxel size: underestimation occurs in areas with crossing fibers. American Journal of Neuroradiology 28, 1102-1106.

O'Sullivan, M., Morris, R.G., Huckstep, B., Jones, D.K., Williams, S.C., Markus, H.S., 2004. Diffusion tensor MRI correlates with executive dysfunction in patients with ischaemic leukoaraiosis. Journal of Neurology, Neurosurgery \& Psychiarty 75, 441-447.

Pearson, R.C., Esiri, M.M., Hiorns, R.W., Wilcock, G.K., Powell, T.P., 1985. Anatomical correlates of the distribution of the pathological changes in the neocortex in
Alzheimer disease. Proceedings of the National Academy of Science of the United States of America 82, 4531-4534.

Perry, R.J., Hodges, J.R., 1999. Attention and executive deficits in Alzheimer's disease. A critical review. Brain 122, 383-404.

Petersen, R.C., Doody, R., Kurz, A., Mohs, R.C., Morris, J.C., Rabins, P.V., Ritchie, K., Rossor, M., Thal, L., Winblad, B., 2001. Current concepts in mild cognitive impairment. Archives of Neurology 58, 1985-1992.

Pierpaoli, C., Jezzard, P., Basser, P.J., Barnett, A., Di Chiro, G., 1996. Diffusion tensor MR imaging of the human brain. Radiology 201, 637-648.

Rogers, J., Morrison, J.H., 1985. Quantitative morphology and regional and laminar distributions of senile plaques in Alzheimer's disease. Journal of Neuroscience 5, 2801-2808.

Roman, G.C., Tatemichi, T.K., Erkinjuntti, T., Cummings, J.L., Masdeu, J.C., Garcia, J.H., Amaducci, L., Orgogozo, J.M., Brun, A., Hofman, A., Moody, D.M., O'Brien, M.D., Yamaguchi, T., Grafman, J., Drayer, B.P., Bennett, D.A., Fisher, M., Ogata, J., Kokmen, E., Bermejo, F., Wolf, P.A., Gorelick, P.B., Bick, K.L., Pajeau, A.K., Bell, M.A., DeCarli, C., Culebras, A., Korczyn, A.D., Bogousslavsky, J., Hartmann, A., Scheinberg, P., 1993. Vascular dementia: diagnostic criteria for research studies. Report of the NINDSAIREN International Workshop. Neurology 43, 250-260.

Rosen, H.J., Gorno-Tempini, M.L., Goldman, W.P., Perry, R.J., Schuff, N., Weiner, M., Feiwell, R., Kramer, J.H., Miller, B.L., 2002. Patterns of brain atrophy in frontotemporal dementia and semantic dementia. Neurology 58, 198-208.

Schmierer, K., Wheeler-Kingshott, C.A., Tozer, D.J., Boulby, P.A., Parkes, H.G., Yousry, T.A., Scaravilli, F., Barker, G.J., Tofts, P.S., Miller, D.H., 2008. Quantitative magnetic resonance of postmortem multiple sclerosis brain before and after fixation. Magnetic Resonance Medicine 59, 268-277.

Selden, N.R., Gitelman, D.R., Salamon-Murayama, N., Parrish, T.B., Mesulam, M.M., 1998. Trajectories of cholinergic pathways within the cerebral hemispheres of the human brain. Brain 121, 2249-2257.

Sjöbeck, M., Haglund, M., Englund, E., 2006. White matter mapping in Alzheimer's disease: a neuropathological study. Neurobiology of Aging 27, 673-680.

Tomimoto, H., Ohtani, R., Shibata, M., Nakamura, N., Ihara, M., 2005. Loss of cholinergic pathways in vascular dementia of the Binswanger type. Dementia and Geriatric Cognitive Disorders 19, 282-288.

Van Straaten, E.C., Scheltens, P., Knol, D.L., Van Buchem, M.A., Van Dijk, E.J., Hofman, P.A., Karas, G., Kjartansson, O., De Leeuw, F.E., Prins, N.D., Schmidt, R., Visser, M.C., Weinstein, H.C., Barkhof, F., 2003. Operational definitions for the NINDS-AIREN criteria for vascular dementia: an interobserver study. Stroke 34, 1907-1912.

Yamauchi, H., Fukuyama, H., Nagahama, Y., Katsumi, Y., Hayashi, T., Oyanagi, C., Konishi, J., Shio, H., 2000. Comparison of the pattern of atrophy of the corpus callosum in frontotemporal dementia, progressive supranuclear palsy, and Alzheimer's disease. Journal of Neurology, Neurosurgery \& Psychiarty 69, 623-629.

Yoshiura, T. Mihara, F. Ogomori, K., Tanaka, A., Kaneko, K., Masuda, K, 2002. Diffusion tensor in posterior cingulate gyrus: correlation with cognitive decline in Alzheimer's disease. NeuroReport 13, 2299-2302.

Yoshiura, T., Mihara, F., Koga, H., Noguchi, T., Togao, O., Ohyagi, Y., Ogomori, K., Ichimiya, A., Kanba, S., Honda, H., 2006. Cerebral white matter degeneration in frontotemporal dementia detected by diffusion-weighted magnetic resonance imaging. Academic Radiology 13, 1373-1378.

Zhang, Y., Schuff, N., Jahng, G.H., Bayne, W., Mori, S., Schad, L, Mueller, S., Du, A.T, Kramer, J.H., Yaffe, K., Chui, H., Jagust, W.J., Miller, B.L., 2007. Diffusion tensor imaging of cingulum fibers in mild cognitive impairment and Alzheimer disease. Neurology 68, 13-19. 